\title{
An Accurate Method for Cloth Digitalization for Virtual Try on
}

\author{
Qingya Zhang \\ Department of Media Arts and Technology \\ Harbin Institute of Technology \\ Harbin, China
}

\author{
Jian Ou \\ Department of Media Arts and Technology \\ Harbin Institute of Technology \\ Harbin, China
}

\author{
Jianyi Wang \\ Department of Media Arts and Technology \\ Harbin Institute of Technology \\ Harbin, China
}

\begin{abstract}
With the development of e-commerce, online shopping becomes more and more important and it is replacing offline shopping. However, cloth try on is still not available in current e-commerce. The key problem for virtual try on is the digitalization of real cloth. Traditional scanning technique like laser scanner or multi camera system can not deal with the complex nature of cloth, such as seams and is difficult to scan the interior of cloth. And the scanning cloth is not suitable for physics simulation because of the complex topology of mesh cloth. We develop a new way to scan the real cloth in a traditional cloth model method by lay out the cloth with sewing connection. This method captured the exact structure of cloth, and the simulation for cloth try on could be much more efficient and reliable.
\end{abstract}

Keywords—virtual try on; cloth digitalization; online shopping

\section{INTRODUCTION}

With the rapid development of e-commerce technology, online shopping has become a major sales channel for the apparel industry. It is a pity that existing clothes online shopping platforms usually rely on twodimensional pictures to display clothing styles for the customers, and it is difficult for customers to accurately know whether the clothing fit well, or collocation is consistent with their own styles. Size does not match, color, style is not suitable and other issues, making the exchange, return goods phenomenon occurred frequently, which become the bottleneck of the development of online apparel industry.

At the same time, in recent years, online virtual try on develops rapidly, it changes people's traditional try on concepts, and bring a lot of convenience in the meantime. Users only input their own body data, the system will match the corresponding virtual model based on the user's 3D data. The users can choose different styles and sizes of clothing to have a try. The method does have great improvement compared with the traditional way of photos, but due to the limitations of existing technology, users are still not sure whether the clothing styles are consistent with their own style, or whether it can meet the user's expected effect. As a key

The Interactive Mirror Project is sponsored by National Science and technology support program project in 2015 (project number: 2015BAH31F01). technique of virtual try on system, the development of 3D data acquisition and motion capture technology requires the characters of rapid, accurate and real-time.

\section{CURRENT DIGITALIZATION TECHNIQUES}

$3 \mathrm{D}$ clothes data is mostly in online shopping [2]. Being limited to current network and $3 \mathrm{~d}$ model complexity, current simulation for clothes doesn't meet the need in online environment. Most of the Cloth simulation is based on $2 \mathrm{~d}$ [3].There are two ways of simulation: Geometry based clothes simulation, physics based simulation. Geometry based method required creating cloth patches and cloth curves to generate clothes model, it is intuitive and fast. Physics based method can simulate clothes dressed on human body; however, the scanning process is complicated and can not provide the seam information.

3D Scanning techniques is very common in industry design, medical study, Digital Content creation and cloth design [5]. When we want to digitalize the real world object, we use the 3D scanning technique. Most of the technique capture the real world object in point cloud data [6], then point cloud data could be used to generate the mesh data. However, when the $3 \mathrm{~d}$ scanning technique comes to cloth digitalization. Here is the $3 \mathrm{~d}$ scanning result of a cloth "Fig. 1".

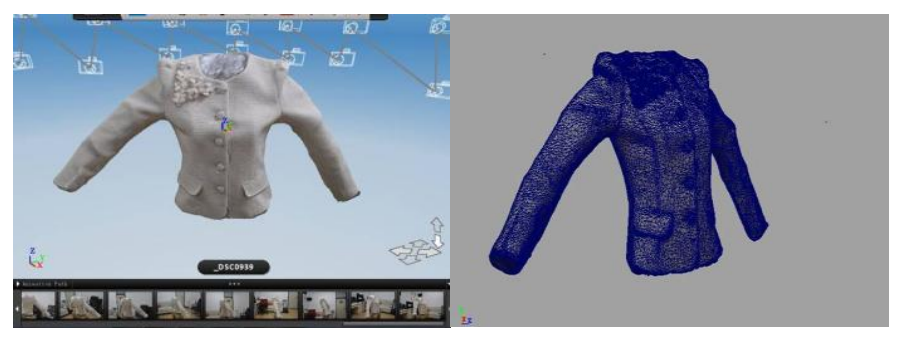

Fig. 1. Cloth scanning using multi camera scanning system

Although the point cloud scanning technique could scan every details of the cloth, the seams, the buttons, and the 
decoration. There are obvious drawbacks: Firstly, the important topology is lost, the scanning system treats the seams as point connection. We couldn't figure how patches of the cloth are connected each other. Secondly, separate components are scanned as meshes that can't be separated in post processing work. Finally, the meshes are not suitable for dynamic simulation for cloth because some computing dynamic algorithm is based on layout $2 \mathrm{~d}$ cloth patches [7].

To solve this problem, we develop a method by first measuring the seam lines, and then layout the cloth patches in $2 \mathrm{~d}$ spaces.

Our paper focuses on the digitalization pipeline of cloth, explain the basic methods of scanning a cloth "Fig. 2".

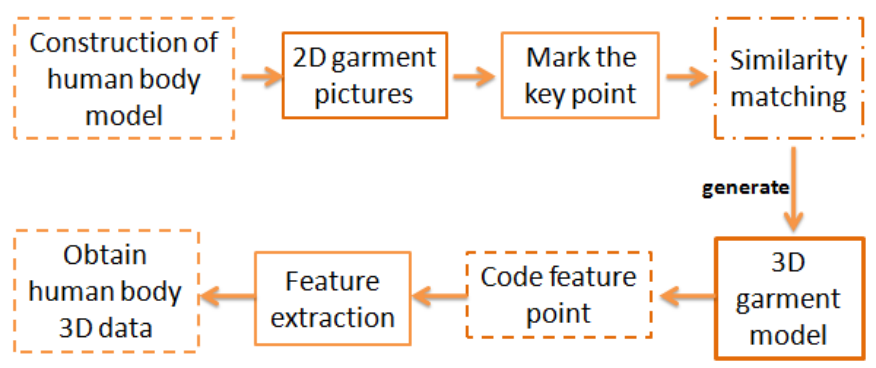

Fig. 2. Pipeline of Clothes Digitalization

\section{IMPLEMENTATION}

\section{A. Cloth Pattern Acquisition}

In clothes design and tailoring, "datum line" is the foundation for tailoring. We must first get the datum line before we get the pattern. Clothes datum line is index line on the human model [8]. There are 2 kinds of index line, which go vertically or horizontally. In a classical suit, the vertical base lines composed of forward/backward center lines, sidelines, and the backward/forward princess line. The horizontal lines consist of the Bust line, waist line and hip line. The datum lines divide the clothes into several patches. The frontal Clothes is divided into left and right patches. On the Front right part, the bust line, waist line, hip line, center line and princess line divided the clothes into 6 patches, so is the left counterpart. The front suit is divided into 12 patches which are symmetrical. The back of suit is also divided into 12 symmetrical patches. The suit consists of 24 patches "Fig. 3". For the trouser, the front is divided into 12 patches, and the back is divided into 24 patches.
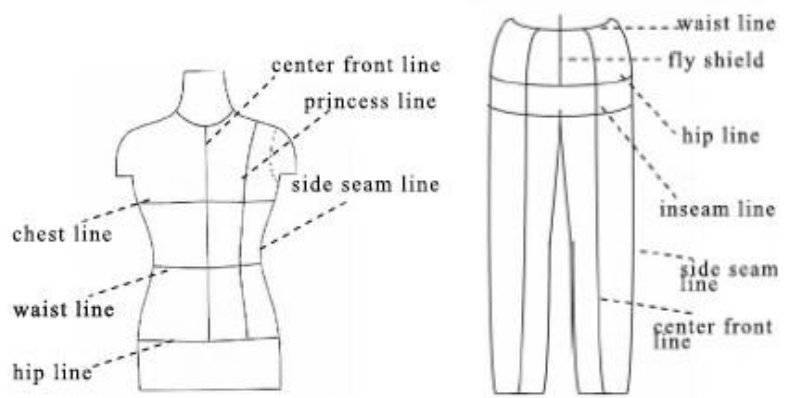

Fig. 3. Key Point Extractions
The patches have regular and smooth shapes, so it is easy to depict the outline of the patches with discreet key feature point. There are 2 ways of drawing the feature point of the patches. The first one disassemble the clothes into its original separate patches, and scans the patches into computer. Then in Marvelous Designer, we mark them by hand. The second solution is to layout the cloth on table, take pictures from different angles, and mark the patches key point. Comparing the two techniques, we found that although the first one destroys the original cloth, but its scan result is much better than the later [9].

\section{B. Sewing and Connection}

The patches are in $2 \mathrm{D}$ spaces in order to recreate the clothes. We need to sew the patches in $3 \mathrm{~d}$ space, and turned the $2 \mathrm{~d}$ meshes into $3 \mathrm{~d}$ meshes.

Firstly, we need to place each patch at its desired 3d location. And then we make connection links between patches. The links are indicated as yellow dotted lines "Fig. 4".

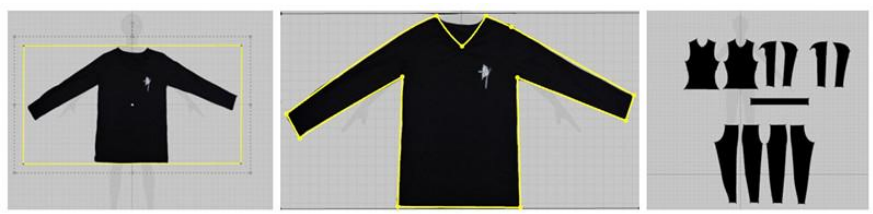

Fig. 4. Cloth Sewing and Connection

\section{Simulation}

The clothes final shape is simulated against an avatar, and simulation here is using multi spring system. "Fig. 5" There are three kinds of springs: the stretch spring, the shear spring and the bend spring. The stretch spring keeps the length between every point, this preserve the total area of the cloth, spring between p0 and p1. "Fig. 6" The shear spring keeps the angles within a polygon, and keeps the polygon from distortion [10]. Usually the shear spring connect vertices at the diagonal, see "Fig. 6" spring connecting $\mathrm{p}_{0}$ and $\mathrm{p}_{4}$. And finally the bend spring will preserve the tension force inside the clothes; it keeps the cloth from bending. Usually the bend spring connects every other point. For example, bending spring between $\mathrm{p}_{0}$ and $\mathrm{p}_{2}$ will keep the angle of line $\mathrm{p}_{0} \mathrm{p}_{1}$ and line $\mathrm{p}_{1} \mathrm{p}_{2}$.Bending spring between $\mathrm{p}_{0}$ and $\mathrm{p}_{2}$ will keep the angle of line $\mathrm{p}_{0} \mathrm{p}_{4}$ and line $\mathrm{p}_{4} \mathrm{p}_{8}$.

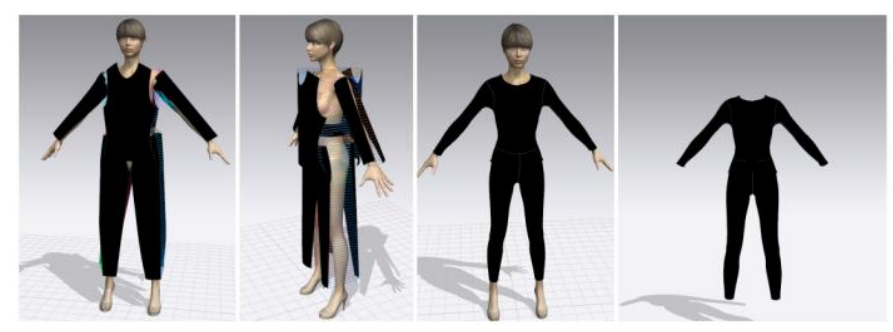

Fig. 5. Final Simulation Result 


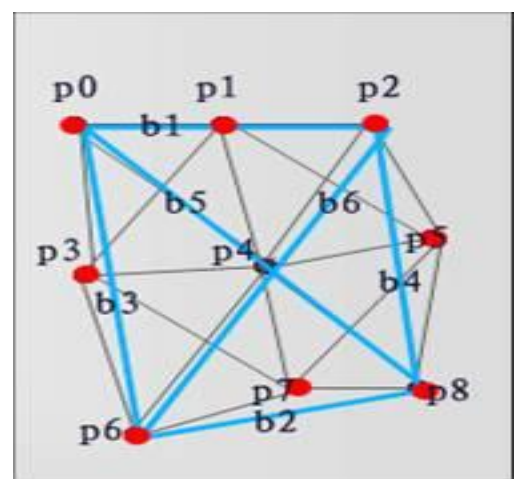

Fig. 6. Cloth Sewing and Connection

The seams between patches are stitched using springs too, which will connect the patches together. "Fig. 7" However direct connection might cause sharp edge at the sin, which is not we are expecting. In order to make connection smooth and nice, we need 1 st order continuity, and for the curvature smooth and nice, we need 2nd order continuity. Spring S0,S1,S2 keep 1st order continuity, Spring C0,C1,C2 keeps 2nd order continuity[11].

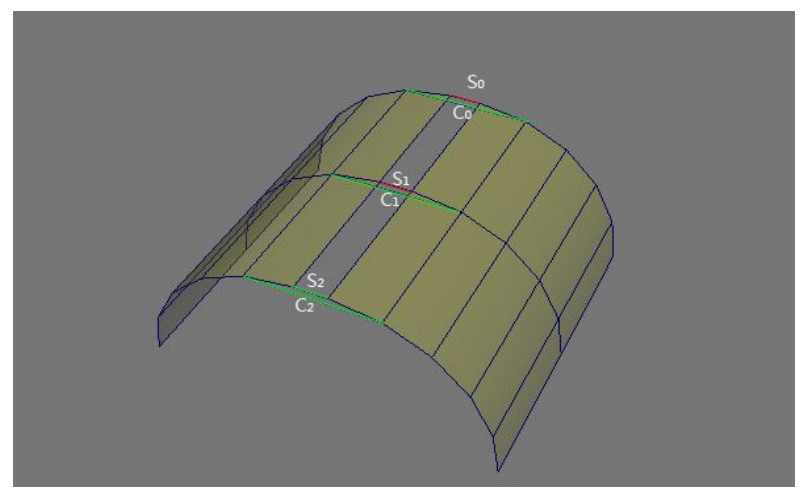

Fig. 7. Seams stitching

The collision between the avatar and clothes patches simply point face intersection test. To make it run faster, we take advantages of NVIDIA physics SDK.

\section{FUTURE WORK}

Our method could digitalize simple thin clothes, and it turns out to be exact the same as the real one. However, the clothes are various and might have more complex structure, such as multiple layers, and patches could not be treated as thin planes. To deal with more complex structure, we need a better algorithm for collision between layers of cloth. And for the thick clothes simulation, we need to constraint exterior layer to the inner layer of clothes, and we have to calculate the complex intersection between clothes. For more natural simulation, we need to consider more parameters like Poisson ratio.

\section{CONCLUSION}

Virtual try on is getting more and more interest in recent years, because online clothes shopping couldn't provide a good selection for the customers. Clothes returns are very common in online shopping; it takes too much expense for the business and customers. To solve this problem, we have to establish a library of digital clothes. In this paper, we present a feasible and accurate clothes scanning technique which can be used in clothes digitalization.

\section{REFERENCES}

[1] Choi K J, Ko H S.Research problems in clothing simulation [J]. Computer Aided Design, 2005, 37(6): 585592

[2] Chittaro L, Corvaglia D.3D virtual clothing: from garment design to web $3 \mathrm{~d}$ visualization and simulation $[\mathrm{M}]$. Proceedings of the eighth internatio nal conference on 3D Web technology. Saint Malo, France; ACM. 2003: $73 \mathrm{ff}$

[3] Meng Y W, Mok P Y, Jin X G. Interactive virtual try-
on
clothing design
systems
[J]. Compute Aided Design, 2010, 42(4): 310321

[4] Leong I F, Kuo J K, Fang J J. A Clothing Simulation System for Realistic Clothing and Mannequin[J].Computer-Aided Design\& Applications, 2011, 8(3):335-344.

[5] Lu L, Choi Y K, Wang W P, et al. Variational 3D shape segmentation for bounding volume computation[J]. Euro graphics, 2007, 26(3): 329 338 .

[6] Sun Xiaodong, Research on several problems in point cloud data processing of human body[D]. Beijing:Beijing University of technology,2012.

[7] Zheng C. Cloth simulation by is geometric analysis[J]. 2013.MLA.

[8] Werner H M, Thalmann N M, Thalmann D. User interface for fashion design[C].Proceedings on Computer Graphics, North-Holland, 1993:197-204.

[9] Zhang Jun, garment CAD research in the area searching for pieces of suture techniques and implementation[D]. Zhejiang:Zhejiang University, 2006: 10-30 1010-1013.

[10] Sun Minghui, Tang Minghao.Reusable information acquisition and character modeling maneuver based on a dress pattern[J]. Computer \& Telecommunication, 2007

[11] McCartney J, Hinds B K, Seow B L, et al. Dedicated 3D CAD for garment modeling[J]. Journal of Materials Processing Technology, 2000 107(11): 31-36. 